\title{
A new approach for developing food frequency questionnaires: the Food Questionnaire Creator
}

\author{
J. Hooson, M. Warthon-Medina, N. Hancock, J.E. Cade* and on behalf of the DIET@NET \\ consortium \\ Nutritional Epidemiology Group, School of Food Science and Nutrition, University of Leeds, Leeds LS2 9JT, UK
}

The number of validated dietary assessment tools that are easily accessible to researchers are limited. Therefore, the DIETary Assessment Tools NETwork (DIET@NET) project aims to develop a central resource that will provide access to validated dietary assessment tools, and provides a structured system that will enable the creation of online questionnaires.

The development of this online platform was overseen by the DIET@NET partnership that appraised commonly used questionnaires to collect dietary data. The selection of questionnaires was part of a systematic review of systematic reviews ${ }^{(1)}$ which identified validated dietary assessment tools.

The result of this evaluation was the creation of an online platform called the 'Food Questionnaire Creator (FQC)', which will be accessible on the Nutritools website, www.nutritools.org, a central resource for researchers undertaking studies which require dietary measurement. The FQC hosts a number of existing validated food questionnaires which have been transformed and updated from paper-based to web-based. The FQC will also enable researchers to generate new online questionnaires or adapt existing tools; set up a project and send the questionnaire to participants for completion either on-line or paper based; provide detailed nutrient outputs. To use the FQC the relevant foods or combinations of foods must be added; frequency of consumption and information on food portions. Foods will be mapped to the latest UK food composition database. We aim to link the FQC with the National Diet and Nutrition Survey to generate recommended foods contributing to a particular nutrient of interest to include in a questionnaire. All newly developed FQC questionnaires can be made public. Although, currently the FQC only provides UK dietary assessment tools and databases, it has been developed to allow for international databases and tools to be added.

The FQC provides researchers with a range of validated paper-based dietary assessment tools online. The FQC enables researchers to generate reliable food questionnaires. We encourage researchers to make their dietary assessment tools more accessible to others, leading to better consistency and comparability of research in nutrition.

This project was funded by the Medical Research Council (MRC) (ref: MR/L02019X/1).

1. Hooson J, Hancock N, Greenwood DC et al. (2016) Proc Nutr Soc 75(OCE3) 